\title{
Response to La Fountaine et al.
}

\author{
Michael D. Stillman ${ }^{1,2} \cdot$ Steve R. Williams ${ }^{1}$
}

Received: 18 August 2018 / Accepted: 19 August 2018 / Published online: 26 September 2018

(C) International Spinal Cord Society 2018

La Fountaine et al. [1] recently compared the lipid profiles of 401 persons with chronic spinal cord injury (SCI) with those of 197 able-bodied controls. In so doing, they found that adverse serum triglyceride (TG) concentrations in people with SCI are lower than in those without, and reiterated previous insights into links between sympathetic nervous system input and circulating lipid levels.

While this article is well reasoned-and each of its authors has substantively contributed to the physiatric literature-the reader is left questioning its clinical relevance. In concluding that clinicians should "consider instituting appropriate dietary, exercise, and/or pharmacological interventions" in patients with elevated TG concentrations, the authors obscure the fact that none of these has been shown to reduce atherosclerotic burden, risk of major adverse coronary events, or cardiac or overall mortality in people with SCI. There are simply no data upon which to base these recommendations.

Further, while the authors rightly point to recent guidelines reaffirming that low high-density lipoprotein levels are a major risk factor for cardiovascular disease (CVD) and that TG levels $\geq 200$ may identify individuals at increased risk (Grade B recommendation) [2], it is not clear what to do with this information. A review of the use of fibrates for primary prevention of CVD revealed a modest benefit over placebo for combined endpoints, but none for overall or non-CVD mortality [3]. A separate review of the efficacy of niacin with or without the addition of a statin demon-

Michael D. Stillman

michael.stillman@jefferson.edu

$\triangle$ Steve R. Williams

steve.r.williams@jefferson.edu

1 Department of Rehabilitation Medicine, Sidney Kimmel Medical College of Thomas Jefferson University, Philadelphia, USA

2 Department of Internal Medicine, Sidney Kimmel Medical College of Thomas Jefferson University, Philadelphia, USA strated no reduction in overall mortality, cardiovascular mortality, or fatal or non-fatal myocardial infarctions when compared with placebo [4].

While La Fountaine et al.'s article is cleanly written and argued, it underscores the need for a certain aggression in tackling cardio-metabolic disease in SCI. Rather than descriptive analyses of lipid profiles or discussion of how cardiovascular risk factors cluster in people with injuries [5], we need trials of interventions with hard and tangible outcomes. As CVD emerges as the leading cause of mortality among individuals with SCI, what can and ought we do to improve their health and longevity?

\section{Compliance with ethical standards}

Conflict of interest The authors declare that they have no conflict of interest.

\section{References}

1. La Fountaine MF, Cirnigliaro CM, Hobson JC, et al. Establishing a threshold to predict risk of cardiovascular disease from the serum triglyceride and high-density lipoprotein concentrations in persons with spinal cord injury. Spinal Cord. 2018. https://doi.org/ 10.1038/s41393-018-0187-7.

2. Jellinger PS, Handelsman Y, Rosenblit PD, Bloomgarden ZT, Fonseca VA, Garber AJ, et al. American association of clinical endocrinologists and american college of endocrinology guidelines for management of dyslipidemia and prevention of cardiovascular disease. Endocr Pract. 2017;23(Suppl 2):1-87. https://doi.org/10. 4158/EP171764.APPGL.

3. Jakob T, Nordmann AJ, Schandelmaier S, Ferreira-Gonzalez I, Briel M. Fibrates for primary prevention of cardiovascular disease events. Cochrane Database Syst Rev. 2016;11:CD009753 https://doi.org/10.1002/14651858.CD009753.

4. Schandelmaier S, Briel M, Saccilotto R, Olu KK, Arpagaus A, Hemkens LG, et al. Niacin for primary and secondary prevention of cardiovascular events. Cochrane Database Syst Rev. 2017;6: CD009744 https://doi.org/10.1002/14651858.CD009744.

5. Libin A, Tinsley EA, Nash MS, Mendez AJ, Burns P, Elrod M, et al. Cardiometabolic risk clustering in spinal cord injury: results of exploratory factor analysis. Top Spinal Cord Inj Rehabil. 2013;19(3):183-194. https://doi.org/10.1310/sci1903-183. 